\title{
Relation of Anthropometric Measures and Insulin Resistance with Antimullerian Hormone in Premenopausal Women
}

\author{
Eman H El-Adawy ${ }^{1 *}$, Maha M Elshafe ${ }^{1}{ }^{1}$, Mohamed Sherif Abd \\ Elgawad $^{1}$, Soma sheriff Abd EL Gawad ${ }^{2}$ \\ ${ }^{1}$ Internal Medicine Department, Specialized Medical Hospital, Faculty of Medicine, \\ Mansoura University, Mansoura, Egypt \\ Research Article \\ Volume 3 Issue 1 \\ Received Date: December 21, 2017 \\ Published Date: January 26, 2018 \\ DOI: $10.23880 /$ doij-16000172
}

${ }^{2}$ Clinical Pathology Department, Faculty of Medicine, Mansoura University, Mansoura, Egypt

*Corresponding author: Eman $\mathrm{H}$ El-Adawy, Department of Internal Medicine, Endocrinology Division, Specialized Medical Hospital, Mansoura University, Faculty of Medicine, Mansoura, Egypt, Email: eman.eladawy@yahoo.com

\section{Abstract}

Background: It has been suggested that obesity is associated with decreased level of antimullerian hormone (AMH) which considered as a good marker of ovarian reserve.

Aim: The aim is to evaluate the association between obesity and AMH and whether there is relation of the anthropometric measures and insulin resistance with the level of AMH in Egyptian premenopausal women.

Subjects and Methods: Eighty premenopausal women with BMI more than 30 (obese group) and 80 age-matched healthy lean women (control group). BMI, waist circumference (WC), blood pressure (BP) were measured. Fasting blood glucose (FBS), fasting insulin (FI), insulin resistance (HOMA-IR), high sensitive C-reactive protein (hs-CRP) and AMH were analyzed.

Results: AMH levels in obese group were significantly lower than control group. There were significant negative correlations between each of BMI, WC, FBG, hs-CRP, FI and HOMA-IR with AMH $(r=-0.214,-0.226,0.141,-0.264,-0.241$ and -0.258 respectively) (all $p$ values $\leq 0.05$ ). With forward stepwise linear regression analysis we found that HOMA-IR was significantly and independently related to AMH; ( $\mathrm{B}=-0.172$; 95\% CI -0.273: -0.071).Furthermore, HOMA-IR was confirmed to be an independent predictor of $\mathrm{AMH}$ after adjustment of age and BMI; (B = - 0.173; 95\% CI - 0.274; - 0.072) and also by adjustment of age and WC; $(B=-0.13595 \% C I-0.268 ;-0.001)$.

Conclusion: Obesity and insulin resistance are associated with decreased ovarian reserve among Egyptian premenopausal women.

Keywords: Anti-mullerian hormone; Obesity; Ovarian Reserve; Premenopausal Women

\section{Introduction}

Obesity is a worldwide epidemic problem with higher prevalence rate in women [1]. It is associated with the risk of cardiovascular disorders [2], diabetes mellitus [3], stroke [4] and other co morbidities. Moreover, obesity has 


\section{Diabetes and Obesity International Journal}

an impact on reproductive health in women, where it is linked to low rate of pregnancy [5-8] and poorer outcomes in vitro fertilization (IVF) [9]. Moreover, in comparison to lean women, those with obesity have decreased responses to medications of fertility, fewer number of oocytes retrieved [10], and higher risk of maternal and infant complications [11].

Antimullerian hormone (AMH) is a peptide hormone related structurally to inhibin hormone and considered as a member of the transforming growth factor-beta family [12]. It is also known as mullarian inhibiting factor which has a role in sex differentiation in male, while in females it is secreted postnatal by the ovarian follicles, hence It has an important role in folliculogenesis $[13,14]$. It is unlike other ovarian tests not change significantly throughout menstrual cycle14), otherwise it is decreased with aging [13]. Some authorities suggest its measurement to assess premature ovarian failure and polycystic ovary syndrome $[13,14]$, moreover, AMH appears to be a strongest and earlier predictor of number of oocytes retrieved during IVF cycle outcome [15].

It has been suggested that obesity is a factor that reduces AMH, however, there are conflicting results concerning this issue as some studies have been reported an inverse relation between obesity and AMH [16-19], while others have failed to clarify such association [2022]. Because of epidemic prevalence of obesity in Egyptian women and the discrepancies in literatures concerning the association between obesity and $\mathrm{AMH}$, the aim of our study is to investigate the relation between obesity and $\mathrm{AMH}$ and whether there is correlation of obesity related components such as BMI, WC, FBG, hsCRP and insulin resistance with AMH levels among Egyptian premenopausal women.

\section{Methods}

\section{Subjects}

A total of 160 women were included in this study at the age of reproduction (25 to 35); 80 with BMI more than 30 (obese group) and 80 lean females (control group). Obese premenopausal women were recruited from Obesity Clinics in Diabetes and Endocrinology Unit at Specialized Medical Hospital, Mansoura University, Faculty of Medicine, Mansoura City, Egypt, during the period from November 2016 to December 2017. Healthy controls were recruited from the same locality and with the same exclusion criteria of the study group. Informed consents were obtained from all women before they participated in the study, which was approved by the ethical committee of our institution. The inclusion criteria were premenopausal women with regular menstrual cycles for previous three months. Exclusion criteria were current use of OCPS or drugs that may affect ovulation, smoking, pregnancy, lactation, thyroid disorders, previous ovarian or uterine surgery and clinical or ultrasonic criteria of polycystic ovary.

\section{Medical History and Physical Examination}

All participants were subjected to thorough history taking and clinical examination. Anthropometric measures including height, weight, body mass index (BMI) and waist circumference (WC) were obtained using standardized techniques; height was measured to the nearest $0.5 \mathrm{~cm}$, body weight was measured to the nearest $0.1 \mathrm{~kg}$, BMI was calculated as weight in kilograms divided by height in meters squared $(\mathrm{kg} / \mathrm{m} 2)$, WC was measured at the highest point of the iliac crest. Blood pressure was taken in the sitting position after 10 minutes rest using a random-zero sphygmomanometer.

\section{Biochemical Assay}

Fasting serum samples were used for FBG level measurements according to the method of Trinder [23]. Determination of serum insulin was performed using ELISA kit, according to the method of Hwang, et al. [24]. The kit was provided from Diagnostic System Laboratories. Inc. Corporate Headquarters, 445 Medical center Blvd. Webster, Texas 77598-4217 USA.

Insulin sensitivity in the fasting state was assessed with homeostasis model assessment (HOMA) and calculated with the following formula: fasting plasma glucose $(\mathrm{mmol} / \mathrm{L}) \times$ fasting serum insulin $(\mu \mathrm{U} / \mathrm{mL})$ divided by 22 , as described by Matthews, et al. [25] high HOMA scores denotes insulin resistance. The plasma samples were stored at -20C until used for the followings: Quantitative determination of hs-CRP was performed according to the method of Muller, et al. [26] using kits purchased from spin react, S.A. (Saint Estere de bas, Spain).

\section{Hormonal Assay}

Serum AMH was measured using the pico AMH ELISA (lower limit of detection $0.0016 \mathrm{ng} / \mathrm{mL}, 1 \mathrm{ng} / \mathrm{mL} 57.14$ pmol/L) (Ansh Labs, Webster, TX). Samples were run at a $1 / 10$ dilution, then neat for the samples that were low. $\mathrm{AMH}$ values that were below the lower limit of detection (n 5 3) were assigned a value of $0.0011 \mathrm{ng} / \mathrm{mL}$ using an established formula [27]. The AMH ELISA has intra- and inters assay coefficients of variation (CVs) of $6.9 \%$ and $6.5 \%$, respectively. 


\section{Diabetes and Obesity International Journal}

\section{Statistical Analysis}

All analysis were done using SPSS version 20 for windows. A tailed P value less than 0.05 was considered significant. Continuous data presented as mean \pm SD. Comparative analysis between two study groups was done using Student st- test. All correlations were done using person correlation test. Association between clinical and biochemical parameters of obesity and AMH was done using forward stepwise linear regression analysis using predictors that found to be significantly related to $\mathrm{AMH}$ in correlation tests. Association between each of FBG, FI and HOMA-IR with AMH was done in other linear regression models after adjustment of age, BMI and WC.

\section{Results}

Table 1 illustrates that BMI, WC, hs-CRP, FI and HOMAIR were significantly higher in obese group than control.
While AMH level was found to be significantly lower in obese women than the non- obese ( $p$ value $<0.05$ ). There was a significant negative correlation between each of BMI, WC, hs-CRP, FI and HOMA-IR as predictors and AMH as an outcome (all p value $<0.05$ ), Table 2 .

By using BMI, WC, hs-CRP, FI and HOMA-IR as outcome, forward stepwise linear progression analysis showed that only HOMA-IR was significantly and independently related to AMH (B 95\%CI) $=3.4(-1.2-.3) \quad \mathrm{P}$ value $=0.001$. Table 3 demonstrates that there is no significant association between FBG and AMH even after adjustment of age and body fat components (BMI and WC), (all $\mathrm{p}$ values $\leq 0.05$ ). There is a significant negative association between FI and $\mathrm{AMH}$ after adjustment of age and BMI; however this association became insignificant after adjustment of $W C$ ( $p$ value $\geq 0.05$ ). HOMA-IR is found to be a significant negative predictor of AMH after adjustment of age and body fat parameters (either BMI or $W C$ ), ( $p$ value $\leq 0.005$ ).

\begin{tabular}{|c|c|c|c|}
\hline Parameters & Obese group NO $\mathbf{8 0}$ & Control group NO $\mathbf{4 0}$ & P Value \\
\hline Age (years) & $31.1 \pm 4.5$ & $31.1 \pm 3.1$ & 0.569 \\
\hline S.B.P. $(\mathrm{mmHg})$ & $115.8 \pm 14.2$ & $117.3 \pm 15.2$ & 0.281 \\
\hline D.B.P. $(\mathrm{mmHg})$ & $75.6 \pm 9.0$ & $74.4 \pm 9.5$ & 0.444 \\
\hline BMI $(\mathrm{kg} / \mathrm{m} 2)$ & $36.1 \pm 3.3$ & $21.7 \pm 1.8$ & $<0.001$ \\
\hline W C & $104.2 \pm 14.5$ & $86.3 \pm 5.5$ & $<0.001$ \\
\hline F.B.G mg/dl & $99.3 \pm 15.1$ & $95.6 \pm 9.3$ & 0.067 \\
\hline hs-CRP & $3.6 \pm 1.1$ & $2.3 \pm 0.7$ & $<0.001$ \\
\hline FI (miu/l) & $11.9 \pm 3.6$ & $6.7 \pm 1.2$ & $<0.001$ \\
\hline HOMA-IR & $1.9 \pm 0.8$ & $1.5 \pm 0.3$ & $<0.001$ \\
\hline AMH(ng/ml) & $2.6+0.7$ & $3.2+0.8$ & $<0.001$ \\
\hline
\end{tabular}

Table 1: Characteristics of study groups

Values expressed as means \pm SD.

$*$ p significant $<0.05$.

\begin{tabular}{|c|c|c|}
\hline Predictors & r & P \\
\hline BMI (kg/m2) & -0.214 & 0.007 \\
\hline W C & -0.226 & 0.004 \\
\hline FBG (mg/dl) & -0.141 & 0.075 \\
\hline hs-CRP & -0.264 & 0.001 \\
\hline FI & -0.241 & 0.002 \\
\hline HOMA & -0.258 & 0.001 \\
\hline
\end{tabular}

Table 2: Correlation between AMH and other variables

$\mathrm{P} \leq 0.05$, significant correlation

\begin{tabular}{|c|c|c|}
\hline Predictors & B (95\% CI ) & P value \\
\hline F B G & & \\
- Mode 1 & $-0.008(-0.01 \mathrm{~b}: 0.001)$ & 0.075 \\
- Mode 2 & $-0.007(-0.016: 0.001)$ & 0.078 \\
- Mode 3 & $-0.005(-0.014: 003)$ & 0.211 \\
\hline FI & & \\
\hline
\end{tabular}




\section{Diabetes and Obesity International Journal}

\begin{tabular}{|c|c|c|}
\hline - Mode 1 & $-0.043(-0.07:-0.0016)$ & 0.002 \\
- Mode 2 & $-0.043(-0.071:-0.016)$ & 0.002 \\
- Mode 3 & $-0.031(-0.067:-0.005)$ & 0.088 \\
\hline HOMA & $-0.172(-0.273:-0.071)$ & 0.001 \\
- Mode 1 & $-0.173(-0.274:-0.072)$ & 0.001 \\
- Mode 2 & $-0.135(-0.268:-0.001)$ & 0.048 \\
\hline
\end{tabular}

Table 3: Association between metabolic parameters and AMH

- Mode 1; adjusted for age

- Mode 2; adjusted for age and BMI

- Mode 3; adjusted for age and WC

\section{Discussion}

This cross-sectional study was designed to investigate the association between obesity and $\mathrm{AMH}$, a marker of ovarian reserve, and whether various anthropometric measures are correlated with its level.

The main finding in our study was a significantly lower AMH level in the study group of obese premenopausal women than those with normal weight (control group). The results also demonstrate a significant negative correlation between BMI, WC, hs-CRP, FI and HOMA-IR as predictors and $\mathrm{AMH}$ as an outcome (all $\mathrm{p}$ value $<0.05$ ), however, HOMA-IR only is found to be a significant negative predictor of AMH after adjustment of age and body fat parameters (either BMI or WC), ( $p$ value $\leq 0.005$ ).

Many studies supported the effect of increased BMI on $\mathrm{AMH}$ as freeman et al. who showed that level of $\mathrm{AMH}$ decline more than 65\%in obese women [28], also this result parallel those of Skalbaetal and Buyuk et al. who found a significant negative relationship between BMI and level of AMH [29,30]. Moreover, De Pergola, et al. [31] suggested that overweight and obese fertile women have lower serum levels of FSH, LH, inhibin and estradiol in the early follicular phase and this may support the finding of lower levels of AMH. In addition, some researches demonstrated improvement in reproductive function which was associated with significant decrease in level of AMH following 6 months treatment with metformin for weight loss [32].

Our finding as regard relation between BMI and AMH was in contrast to Sahmay, et al. [33] who showed negative but insignificant relation between BMI and AMH levels. Nardo, et al. and Halawaty, et al. have reported insignificant relationship were found between BMI and circulating AMH level in a cross-sectional study that included 100 premenopausal women [34,35]. Indeed, the association between obesity and AMH primarily derived from studies of women at reproduction with polycystic ovary syndrome (PCOS), where it was found that AMH levels did not appear to vary significantly by body size $[36,37]$. However, among control women without PCOS, BMI was inversely associated with AMH levels.

The mechanism underlying the inverse relationship between obesity and AMH is not entirely clear, a number of theories have been proposed to further explain this relationship. It is possible that insulin resistance in individuals with obesity acts on granulosa cells and consequently alters AMH concentration [38]. There may also be lipotoxic effects on the granulosa cells [39]. It is also suggested that leptin and adiponectin are involved in modulating ovarian function as these adipokines play a role in reproductive processes by way of the hypothalamic-pituitary-ovarian axis [38]. Another possibility is that AMH may be metabolized, stored, and cleared in a different way among subjects with obesity [39].

In our study, the etiology for lower AMH levels among the obese group could not be explored; however we support the suggestion that obesity may create an altered follicular environment. Furthermore, this study demonstrate a significant negative correlation between obesity related parameters; BMI, WC, hs-CRP, FI and HOMA-IR as predictors and AMH as an outcome, however, HOMA-IR only is found to be a significant negative predictor of AMH after adjustment of age and BMI and WC.

Current reports about the relation between insulin resistance and AMH levels are conflicting and heterogeneous especially during the premenopausal age, with some indicating presence and others absence of a correlation between both of them. Published studies of insulin resistance and CRP and ovarian markers are largely limited to investigations of PCOS [29].

In line with our findings, Verit, et al. [40] showed that increased CRP levels and decreased insulin sensitivity 


\section{Diabetes and Obesity International Journal}

were associated with diminished ovarian reserve as determined by antral follicle count (AFC) and estrogen level. The mechanisms by which insulin resistance exerts its effect on AMH have not yet been clarified, but because of $\mathrm{AMH}$ is mainly produced in granulosa cells of preantral follicles and small antral follicles making it reasonable to hypothesis that abnormal insulin action is associated with granulosa cell dysfunction and decreased AMH [35]. Overall our findings raise the possibility that high BMI and insulin resistance might be direct determinant of functional ovarian reserve as assessed by AMH during the reproductive years in obese premenopausal women.

\section{Limitations of study}

The cross-sectional design is considered a limitation as it is only allowed for assessment of associations, thus the relationship of $\mathrm{BM}$ and other obesity related components with AMH in reproductive age should be further investigated to gain additional insight into the long-term effects of obesity on AMH.

\section{Conclusion}

This study of premenopausal Egyptian obese women demonstrated that AMH is significantly lower in such population than those with normal weight and insulin resistance is the only independent predictor to AMH.As it has been suggested that AMH is a strong indicator of ovarian function, our findings might indicate that folliculogenesis and/or ovarian reserve may be affected in premenopausal Egyptian women with obesity. However, more investigation is needed to attain clarifications of such relationship.

\section{Conflicts of Interest}

No potential conflict of interest relevant to this article was reported.

\section{Acknowledgments}

The authors thank all sample donors for their participation to this study and all members of the Obesity Outpatient Clinics at Endocrinology Unit, Specialized Medical Hospital, Mansoura, Egypt.

\section{References}

1. Ogden CL, Carroll MD, Fryar CD, Flegal KM (2015) Prevalence of obesity among adults and youth: United States, 2011-2014. NCHS Data Brief 219: 1-8.
2. Flint AJ, Hu FB, Glynn RJ, Caspard H, Manson JE, et al. (2010) Excess weight and the risk of incident coronary heart disease among men and women. Obesity 18(2): 377-383.

3. Kahn SE, Hull RL, Utzschneider KM (2006) Mechanisms linking obesity to insulin resistance and type 2 diabetes. Nature 444(7121): 840-846.

4. Song YM, Sung J, Davey Smith G, Ebrahim S (2004) Body mass index and ischemic and hemorrhagic stroke: a prospective study in Korean men. Stroke 35(4): 831-836.

5. Wise LA, Rothman KJ, Mikkelsen EM, Sorensen HT, Riis A, et al. (2010) An internet-based prospective study of body size and time-to-pregnancy. Hum Reprod 25(1): 253-264.

6. Gesink Law DC, Maclehose RF, Longnecker MP (2007) Obesity and time to pregnancy. Hum Reprod 22(2): 414-420.

7. van der Steeg JW, Steures P, Eijkemans MJ, Habbema JD, Hompes PG, et al. (2008) Obesity affects spontaneous pregnancy chances in sub fertile, ovulatory women. Hum Reprod 23(2): 324-328.

8. Jensen TK, Scheike T, Keiding N, Schaumburg I, Grandjean P (1999) Fecund ability in relation to body mass and menstrual cycle patterns. Epidemiology 10(4): 422-428.

9. Practice Committee of the American Society for Reproductive Medicine (2015) Obesity and reproduction: a committee opinion. Fertil Steril 104(5): 1116-1126.

10. Fedorcsák P, Dale PO, Storeng R, Ertzeid G, Bjercke S, et al. (2004) Impact of overweight and underweight on assisted reproduction treatment. Hum Reprod 19(11): 2523-2528.

11. Wise LA, Rothman KJ, Mikkelsen EM, Sorensen HT, Riis A, et al. (2010) An internet-based prospective study of body size and time-to-pregnancy. Hum Reprod 25(1): 253-264.

12. Broer SL, Eijkemans MJ, Scheffer GJ, van Rooij IA, de Vet A, et al. (2011) Anti-mullarian hormone predicts menopause: along-term follow-up study in normoovulatory women. J Clinical Endocrinol Metab 96(8): 2532-2539. 


\section{Diabetes and Obesity International Journal}

13. Builinger SL, Visser JA, Themmer AP (2002) Regulation of ovarian failure the role of $\mathrm{AMH}$ in production 124: 601-609.

14. Buyuk E, Seifer DB, Illions E, Grazi RV, Lieman H (2011) Elevated body mass index is associated with lower serum anti-mullerian hormone level in infertile women with diminished ovarian reserve but not with normal ovarian researve. Feril Steril 95(7): 23642368.

15. Clark AM, Ledger W, Galletly C, Tomlinson L, Blaney F, et al. (1995) Weight loss result in significant improvement in pregnancy and ovulation rates in an ovulatory obsess women. Hum Reprod 10(10): 27052712.

16. Meden-Vrtovec H (2004) Ovarian aging and infertility. Clin Exp Obst Gynecol 31(1): 5-8.

17. Metwally N, Lit C, Ledger WI (2007) The impact of obesity on ovarian female reproductive function. Obes Rev 8(6): 515-523.

18. Nardo LG, Christodoulou D, Gould D, Roberts SA, Fitzgerald CT, et al. (2007) Anti-mullerian hormone levels and antral follicle count in women enrolled in invitro fertilization cycles: relationship to lifestyle factors, chronological age and reproductive history. Gynecol Endocrinol 23(8): 486-493.

19. Ohgi S, Nakagawa K, Kojima R, Ito M, Horikawa T, et al. (2008) Insulin resistance in oligomenorrheic infertile women with non-polycystic ovary syndrome. Fertility and Sterility 90(2): 373-377.

20. Pasquali R, Cambinei A (2006) Polycystic ovary syndrome: a multifacted disease from adolescent adult age. Ann Ny Accad Sci 1092: 158-174.

21. Piltonen $\mathrm{T}$, Morin-Papunen $\mathrm{L}$, Koivunen $\mathrm{R}$, Perheentupa A, Ruokonen A, et al. (2005) Serum antimullerian hormone level remain high until late reproductive age and decrease during metformin therapy in women with polycystic ovary. Hum Reprod 20(7): 1820-1826.

22. Sahmay S, Usta T, Erel CT, Imammoglu M, Kucuk M, et al. (2012) Is there any correlation between AMH and obesity in premenopausal women? Arch Gynecol Obes 286(3): 661-665.

23. Trinder P (1969) Determination of Glucose in Blood Using Glucose Oxidase with an Alternative Oxygen Acceptor. Ann Clin Biochem 6(1): 24-27.
24. Hwang IS, Ho H, Hoffman BB, Rwaven GM (1987) Fructose induced insulin resistance and hypertension in rates. Hypertension 10(5): 512-516.

25. Matthews DR, Hosker JP, Rudenski AS, Naylor BA, Treacher DF, et al. (1985) Homeostasis model assessment: insulin resistance and ß-cell function from fasting plasma glucose and insulin concentrations in man. Diabetologia 28(7): 412-419.

26. Miller B, Mellor A, Buckley (2013) Total and Differential White Blood Cell Counts, High-Sensitivity $\mathrm{C}$-reactive protein, and the Metabolic Syndrome in Non-Affective Psychoses. Brain Behav Immun 31: 8289.

27. Fréour $T$, Mirallié $S$, Bach-Ngohou $K$, Denis $M$, Barrière $P$, et al. (2007) Measurement of serum AntiMüllerian Hormone by Beckman Coulter ELISA and DSL ELISA: Comparison and relevance in Assisted Reproduction Technology (ART). Clin Chima Acta 375(1-2): 162-164.

28. Freeman EW, Gracia CR, Sammel MD, Lin H, Lim LC, et al. (2007) Association of AMH levels with obesity in late reproductive age women Fertil Steril 879(1): 101-106.

29. Skałba P, Cygal A, Madej P, Dąbkowska-Huć A, Sikora J, et al. (2011) Is the plasma anti-mullerian hormone level is associated with body weight and metabolic and hormonal disturbances in women with and without polycystic ovary syndrome? Eur J Obstet Gynecol Reprod Biol 158(2): 254-259.

30. Buyuk E, Seifer DB, Illions E, Grazi RV, Lieman H (2011) Elevated body mass index is associated with lower serum anti-mullerian hormone level in infertile women with diminished ovarian reserve but not with normal ovarian reserve. Fertil Steril 95(7): 23642368.

31. De Pergola G, Maldera S, Tartagni M, Pannacciulli N, Loverro G, et al. (2006) Inhibitory effect of obesity on gonadotrophins, estradiole and inhibin $b$ levels in fertile women. Obesity (silver) spring 14(11): 19541960.

32. Fleming R, Harborne L, MacLaughlin DT, Ling D, Norman J, et al. (2005) Metformine reduces serum Mullerian-inhibiting substance levels in women with polycystic ovary syndrome after protracted treatment. Fertil Steril 83(1): 130-136. 


\section{Diabetes and Obesity International Journal}

33. Sahmay $S$, Usta T, Erel CT, Imamoğlu $M$, Küçük $M$ (2012) Is there any correlation between amh and obesity in premenopausal women? Arch Gynecol Obstet 286(3): 661-665.

34. Nardo LG, Christodoulou D, Gould D, Roberts SA, Fitzgerald CT, et al. (2007) Anti-mullerian hormone levels and antral follicle count in women enrolled in in vitro fertilization cycles: relationship to lifestyle factors, chronological age and reproductive history. Gynecol Endocrinol 23(8): 486-493.

35. Halawaty S, El Kattan E, Azab H, El Ghamry N, AlInany $H$ (2010) Effect of obesity on parameters of ovarian reserve in premenopausal women. J Obstet Gynaecol Can 32(7): 687-690.

36. Eldar-Geva T, Margalioth EJ, Gal M, Ben-Chetrit A, Algur N, et al. (2005) Serum anti-mullerian hormone levels during controlled ovarian hyperstimulation in women with polycystic ovaries with and without hyperandrogenism Hum Reprod 20(7): 1814-1819.
37. Fanchin R, Louafi N, Méndez Lozano DH, Frydman N, Frydman R, et al. (2005) per-follicle measurement indicate that anti-mullarian hormone secretion is modulated by the extent of follicular development and lutinization and may reflect qualitatively the ovarian follicular status. Fertile Steril 84(1): 167-173.

38. Hartz AJ, Barboriak PN, Wong A, Katayama KP, Rimm AA (1979) The association of obesity with infertility and related menstrual abnormalities in women. Int J Obes 3(1): 57-73.

39. Weenen C, Laven JS, Von Bergh AR, Cranfield M, Groome NP, et al. (2004) Anti-mullarian hormone expression pattern in human ovary: potential implications for initial and cyclic follicle recruitment. Mol Hum Reprod 10(2): 77-83.

40. Verit FF, Keskin S, Omer B, Yalcinkaya S, Sakar N (2014) Is there any relationship between cardiovascular risk markers and young women with diminished ovarian reserve? Gynecol Endocrinol 30(10): 1-4. 